\title{
Bmi1 cooperates with Dnmt1 associated protein 1 in gene silencing
}

Masamitsu Negishi ${ }^{1,3}$, Atsunori Saraya ${ }^{1}$, Satoru Miyagi ${ }^{1}$, Kenji Nagao $^{4}$, Yoshimasa Inagaki ${ }^{5}$, Mitsuo Nishikawa ${ }^{5}$, Shoji Tajima ${ }^{6}$, Haruhiko Koseki $^{7}$, Hiroshi Tsuda ${ }^{3}$, Yoshinari Takasaki ${ }^{3}$, Hiromitsu Nakauchi ${ }^{2}$, Atsushi Iwama ${ }^{1}$

${ }^{1}$ Department of Cellular and Molecular Medicine, Graduate School of Medicine, Chiba University, Chiba 260-8670, Japan. ${ }^{2}$ Laboratory of Stem Cell Therapy, Center for Experimental Medicine, Institute of Medical Sciences, University of Tokyo, Tokyo 1088679, Japan. ${ }^{3}$ Department of Internal Medicine and Rheumatology, Juntendo University, Tokyo, 113-8421, Japan. ${ }^{4}$ Pharmaceutical Frontier Laboratory and ${ }^{5}$ Pharmaceutical Research Laboratories, Kirin Brewery Co. Ltd., Takasaki, Gunma 370-1295, Japan. ${ }^{6}$ Institute for Protein Research, Osaka University, Suita, Osaka 565-0871, Japan. ${ }^{7}$ RIKEN Research Center for Allergy and Immunology, Laboratory for Developmental Genetics, Yokohama 230-0045, Japan.

Correspondence to: Atsushi Iwama, Department of Cellular and Molecular Medicine, Graduate School of Medicine, Chiba University, 1-8-1 Inohana, Chuo-ku, Chiba, 260-8670 Japan. Tel. 81-43-226-2187; Fax. 81-43-226-2191

E-Mail: aiwama@,faculty.chiba-u.jp 


\begin{abstract}
Polycomb group $(\mathrm{PcG})$ proteins are involved in gene silencing through chromatin modifications. Among polycomb repressive complexes (PRC), PRC1 exhibits H2A-K119 ubiquitin E3 ligase activity. However, the molecular mechanisms underlying PRC1mediated gene silencing remain largely obscure. In this study, we found that Bmil directly interacts with Dnmt1 associated protein 1 (Dmap1), which has been characterized to associate with the maintenance DNA methyltransferase, Dnmt1. Bmil was demonstrated to form a ternary complex with Dmap1 and Dnmt1 with Dmap1 in the central position. Chromatin immunoprecipitations confirmed the ternary complex formation within the context of the PRC1 at the Bmil target loci. Loss of Dmap1 binding to the Bmil target loci was tightly associated with derepressed gene expression in $\mathrm{Bmil}^{-/-}$cells. Dmapl knockdown exhibited the same impact as Bmil knockout did on the expression of Bmi1 targets, including Hox genes. Collectively, our findings suggest that Bmil incorporates Dmap1 in polycomb gene silencing.
\end{abstract}

Key words: Bmi1 / Dmap1 / Dnmt1 / polycomb gene / gene silencing 


\section{Introduction}

Cell-type specific gene expression profiles are stabilized by changes in chromatin structure and DNA methylation patterns. Cellular memory of chromatin modifications can be faithfully maintained through subsequent cell divisions by the counteractions of transcriptional activators of the trithorax group (TrxG) and repressors of the polycomb group (PcG) (1). PcG proteins form multiprotein complexes that play an important role in the maintenance of transcriptional repression of target genes. So far, four major PcG complexes have been identified and well characterized. PRC1 includes Bmil/Pgcf4, Rnf110/Mel18, Phc1/Mph1/Rae28, Cbx2/M33, Scmh1, Ring1a and Rind1b, and PRC2/3 includes Eed, Enx1/Ezh2 and Suz12 $(1,2)$. A transformation-specific PRC4 complex includes Ezh2, Eed isoform 2, Suz12 and the NAD ${ }^{+}$-dependent histone deacethylase SirT1 (3). The PRC1 and PRC2/3/4 complexes possess H2A-K119 ubiquitin E3 ligase activity $(4,5)$ and H3-K27 methyltransferase activity (1), respectively. Both histone modifications contribute to $\mathrm{PcG}$ gene silencing. Although no physical associations have been demonstrated between the two complexes, H3-K27 methylation serves as a binding site for the recruitment of the PRC1 complex. Thus, the two PcG complexes could function in a cooperative manner to maintain gene silencing.

PRC1 has been implicated in stem cell self-renewal $(6,7)$, a process by which epigenetic cellular memory is precisely inherited to daughter cells through cell division. Among PRC1 components, we have demonstrated that Bmil plays a central role in the inheritance of stemness of hematopoietic stem cells in both loss-of-function and gain-of-function analyses $(8,9)$. Bmil in the PRC1 complex contributes to the H2A-K119 ubiquitin E3 ligase activity (5). However, its interacting molecules other than core PRC1 components remain to be determined. 
Negishi M, et al. 4

In this study, we screened novel interacting molecules with Bmil and found that Bmil forms a complex with Dmap1 and the maintenance DNA methyltransferase Dnmt1 at its target loci. We further confirmed the involvement of Dmap1 in gene silencing at the Bmi1 target loci using Dmapl knockdown cells. Our finding unveils a novel aspect of gene silencing mediated by Bmil. 


\section{Materials and Methods}

\section{Yeast two-hybrid screening}

Mouse hematopoietic stem cell (CD34 ${ }^{-} \mathrm{c}-\mathrm{Kit}^{+} \mathrm{Sca}-1^{+}$lineage $\left.{ }^{-}\right)$library (10) was screened using a MATCHMAKER GAL4 Two-Hybrid System 2 (Invitrogen). The transformants were selected on plates lacking histidine, leucine and tryptophan, and then assayed for $\beta$ galactosidase activity.

\section{Immunoprecipitation and GST pull-down}

Transfected COS7 cells and NIH3T3 cells were lysed in hypertonic buffer $\left(20 \mathrm{mM} \mathrm{Na}-\mathrm{PO}_{4}\right.$ at $\mathrm{pH} 7.0,250 \mathrm{mM} \mathrm{NaCl}, 0.1 \% \mathrm{NP}-40,30 \mathrm{mM} \mathrm{Na}_{4} \mathrm{P}_{2} \mathrm{O}_{7}, 10 \mathrm{mM} \mathrm{NaF}, 5 \mathrm{mM}$ EDTA, $1 \mathrm{mM}$ DTT and protease inhibitor cocktails (Roche), and then the immunoprecipitation was performed. For the GST pull-down assay, HA-tagged Dmap1 and myc-tagged Dnmt1 were translated in vitro using a TNT reticulocyte lysate transcription/translation system (Promega) and mixed with GST-Bmil fusion protein. The reaction was carried out at $4{ }^{\circ} \mathrm{C}$ for 2 hours and the beads were washed four times with NETN buffer ( $2 \mathrm{mM}$ Tris-HCl, pH8.0, $1 \%$ NP-40, 0.1\% glycerol, $150 \mathrm{mM} \mathrm{NaCl}, 1 \mathrm{mM}$ EDTA and a proteinase inhibitor cocktail). The antibodies used were a monoclonal anti-Bmil (229F6; Upstate Biotechnology), a polyclonal anti-Dmap1 (ab2848; abcam), a polyclonal anti-Dnmt1 (kindly provided by Dr. S. Tajima), a monoclonal anti-Flag (Sigma), a monoclonal anti-Myc (9E10; Santa Cruz) and a polyclonal anti-HA (Y-11; Santa Cruz) antibody.

\section{Glycerol gradient sedimentation analysis}

$2.5 \times 10^{7}$ NIH3T3 cells were treated on ice for $10 \mathrm{~min}$ in lysis buffer [50mM Hepes (pH7.6), $150 \mathrm{mM} \mathrm{NaCl}, 0.1 \%$ Triton, $1 \mathrm{mM}$ EDTA] and were sonicated sequentially for $10 \mathrm{~min}$ in $15 \mathrm{~s}$ burst with 30 s cooling in between. The whole cell lysate $(0.25 \mathrm{ml})$ was loaded onto a 10.4 $\mathrm{ml}$ of $10-50 \%$ glycerol gradients in $50 \mathrm{mM}$ HEPES (pH 7.6), $150 \mathrm{mM} \mathrm{NaCl}, 1 \mathrm{mM}$ EDTA 
and $0.1 \%$ Triton $\mathrm{X}$, and spun at 38,000 rpm for $13 \mathrm{hr}$. The supernatant were separated into 36 fractions $(250 \mu 1 \mathrm{each})$, which were analyzed by Western blot using anti-Bmi1, Dmap1 and Dnmtl antibodies. To confirm fractionation efficacy, thyroglobulin and aldolase (SIGMA) were fractioned as size markers on a separate gradient that was run in parallel, then subjected to SDS-PAGE followed by coomassie brilliant blue staining analysis.

\section{MS-RDA, RT-PCR analysis and 5-aza-dC treatment}

MS-RDA screening was performed as described (11). Genomic DNA isolated from Lineage ${ }^{-}$immature hematopoietic cells from E14.5 wild-type and Bmi $1^{-/-}$littermate fetal livers were subjected to MS-RDA. Differentially methylated DNA fragments were cloned into a TA vector, sequenced, and their genomic localization determined with a BLAST search. RT-PCR was carried out using normalized cDNA by quantitative PCR using TaqMan rodent GAPDH control reagent (Perkin-Elmer Applied Biosystem). Primer sequences are available on request. MEL cells and MEFs were treated with 5-aza-dC (1 $\mu \mathrm{M}$; Sigma) for 4 days. Lentivirus vectors (CS-H1-shRNA-EF-1 $\alpha$-EGFP) expressing shRNA against murine Dmapl (target sequence: GGCACAGATCTCA AGATAC) and Luciferase were prepared and the viruses were produced as previously described (9).

\section{Chromatin immunoprecipitation}

Cross-linked chromatin was sonicated to generate fragments with an average length of 300$400 \mathrm{bp}$, and was subjected to immunoprecipitation using the following antibodies; anti-Bmi1 and anti-Dmap1; anti-Dnmt1 (H-300), anti-Mel-18 (H-115), anti-H3-acethyl-K9/K14 (sc8655-R), and anti-rabbit IgG (Santa Cruz); anti-Ring1b and anti-Scmh1 (kindly provided by

Dr. H. Koseki); anti-H3-trimethyl K27 and anti-ubiquityl H2A (E6C5)(Upstate Biotechnology). 


\section{Results}

\section{Bmi1 forms a ternary complex with Dmap1 and Dnmt1.}

To identify the proteins that cooperate with Bmil in gene silencing, we performed a yeast two-hybrid screening of a cDNA library prepared from mouse bone marrow hematopoietic stem cells using Bmil lacking the C-terminal Proline/Serine-rich domain as a bait. One positive clone encoded full-length Dmap1, a co-repressor that interacts and co-localizes to DNA replication foci with Dnmt1 throughout the S phase (12) (Fig. 1A). Dmap1 interacts with Dnmtl through its middle portion, which includes the SANT domain. Detailed mapping of interaction regions of the two proteins was carried out using a yeast two-hybrid system. The regions of Bmi1, which include a ring finger domain and helix-turn repeats, respectively, bound to the Dmap1 N-terminus, the region outside the Dnmt1 interaction site of Dmap1 (Fig. 1A). These findings evoked the possibility that Bmil forms a ternary complex with Dmap1 and Dnmt1. To address this issue, we performed a coimmunoprecipitation assay using transfected cells with Flag-Bmi1, HA-Dmap1 and MycDnmt1. Both HA-Dmap1 and Myc-Dnmt1 co-immunoprecipitated with Flag-Bmi1 with an anti-Flag antibody, and both Flag-Bmi1 and Myc-Dnmt1 did so with HA-Dmap1 with an anti-HA antibody (Fig. 1B). The direct interaction of Bmil with Dmap1 was confirmed in GST pull-down assay; whereas, Bmil did not bind to Dnmt1 directly (Fig. 1C). These results indicate that Bmil forms a ternary complex with Dmap1 and Dnmt1 by directly interacting with Dmap1.

The ternary complex formation was further determined in Bmil-transfected NIH3T3 fibroblasts, where endogenous Dmap1 and Dnmt1 co-immunoprecipitated with Bmi1 using an anti-Bmil antibody (Fig. 1D). We next fractionated whole cell lysates derived from untransfected NIH3T3 cells on a glycerol gradient. Importantly, Bmi1, Ring1b, Dmap1 
and Dnmt1 were co-fractionated in high-molecular-weight fractions ( $>1 \mathrm{MDa}$; Fractions 3033)(Fig. 1E). These findings indicate that the ternary complex is formed within the context of the PRC1.

\section{Bmi1 cooperates with Dmap1 and Dnmt1 in gene silencing.}

DNA methylation is an important component of the epigenetic control of gene silencing. DNA methyltransferases, namely Dnmt1, Dnmt3a and Dnmt3b, are required for the establishment and maintenance of genomic DNA methylation patterns. It is widely accepted that Dnmt3a and Dnmt3b function primarily as de novo methyltransferases that establish DNA methylation patterns; whereas, Dnmt1 functions primarily as a maintenance methyltransferase that copies the parental-strand methylation pattern onto the daughter strand after each round of DNA replication. A recent study has implicated Ezh2, a component of PRC2/3/4, in the control of DNA methylation (13). Ezh2 serves as a recruitment platform for DNA methyltransferases, including Dnmt1, Dnmt3a and Dnmt3b, thereby connecting chromatin modifications and DNA methylation. The finding that Bmil forms a complex with Dmap1 and Dnmt1 evoked the possibility that the ternary complex has a role in the control of local DNA methylation. We therefore took a genome-wide screening of $\mathrm{Bmil}^{-/-}$genomic DNA for differentially methylated genes using methylationsensitive-representational difference analysis (MS-RDA)(11). This method enables us to identify DNA fragments differentially methylated between the two DNA sources. MSRDA screening was performed using genomic DNA of fetal liver hematopoietic cells obtained from wild-type and $\mathrm{Bmil}^{-/-}$littermates (E14.5). We isolated a large number of genomic DNA fragments, and 30 of them were randomly sequenced. Among them, we selected 12 fragments located in the putative promoter/enhancer regions for further analysis (Supplementary Table S1). The expression of the 5 genes was depressed in $\mathrm{Bmil}^{-/-}$fetal 
liver hematopoietic cells (Fig. 2A). Furthermore, 3 out of 5 genes $-\mathrm{Clq}$ and TNF related protein 6 (C1qTrO), Ring finger protein 103 (RNF103) and WD repeat domain 77 (WDr77), were clearly reactivated in an immature hematopoietic cell line, MEL, by treatment with the DNA methylation inhibitor 5-aza-deoxycytidine (5-aza-dC) (Fig. 2B), indicating that these genes are sensitive to DNA methylation in its transcriptional repression. Given that the PcG proteins are involved in gene silencing, the derepressed genes might represent direct Bmil targets. We therefore performed detailed analysis on these loci.

We first asked whether Bmil is targeted to the Bmil target loci and forms a ternary complex with Dmap1 and Dnmt1. Chromatin immunoprecipitations (ChIP) using MEL cells confirmed the ternary complex formation within the context of the PRC1 at these loci (Fig. 2C). Of note was that loss of Bmil binding in Bmil knockout fetal liver cells profoundly affected the Dmap1 binding to the same loci and Dnmt1 binding to a lesser extent (Fig. 3). Consistent with an important role for Bmil in H2A ubiquitination, the Bmil knockout resulted in a significant decrease in $\mathrm{H} 2 \mathrm{~A}$ ubiquitination, and in a significant increase in acetylation of $\mathrm{H} 3 \mathrm{~K} 9$, a hallmark of active chromatin (Fig. 3). In contrast, the tri-methylation status of $\mathrm{H} 3 \mathrm{~K} 27$ in the absence of Bmil varied among the analyzed loci.

To evaluate the effect of the ternary complex on the local $\mathrm{CpG}$ methylation status, we next carried out bisulfite genomic sequencing of the MS-RDA fragments at the putative enhancer and promoter regions of $C 1 q \operatorname{Tr} 6$ and $W D r 77$ genes, respectively (Supplementary Fig. S1). Genomic DNA was prepared from mouse fetal liver Lineage immature hematopoietic cells from wild-type and $\mathrm{Bmil}^{-/-}$littermates (E14.5). Interestingly, several CpGs located within analyzed region showed differential methylation patterns between wild-type and $\mathrm{Bmil}^{-/-}$fetal liver hematopoietic cells. The ClqTr6 locus included several hypomethylated CpGs, while the WDr77 locus (7414543-7414663) included both local hypomethylation and hypermethylation. These differential methylation patterns were 
confirmed by repeated experiments using genomic DNA from three different littermates. In contrast, CpGs situated outside the WDr77 MS-RDA fragment region (74144697414543) did not show significant difference in methylation status. These data indicate the involvement of DNA methylation control in gene silencing at the Bmil target loci. However, Bmil deficiency did not substantially alter the quantitative methylation status of both major and minor satellite sequences (Supplementary Fig. S2A).

\section{Hox loci are derepressed in Bmi1 ${ }^{--}$and Dmap1 knockdown MEFs.}

Finally, we examined the role of the Bmil-Dmap1 complex in the transcriptional control of homeobox (Hox) genes. Previous studies in mice indicated that loss of Bmil results in a shift in Hox gene expression boundaries and shows posterior transformation (14). We analyzed the expression of Hox genes that are reportedly derepressed in expression in $\mathrm{Bmil}^{-}$ - mouse embryonic fibroblasts (MEF)(5). In addition to Bmil knockout, we evaluated the role of Dmap1 by using the Dmapl knockdown technique. MEFs were infected with a lentivirus expressing shRNA against Dmap1, which efficiently inhibited Dmap1 expression (Fig. 4A). Although Dmap1 has been reported to co-localize to DNA replication foci with Dnmt1 throughout the S phase (12), Dmapl knockdown as well as Bmil knockout did not significantly alter the quantitative methylation status of both major and minor satellite sequences (Supplementary Fig. S2B). The expression of HoxA7, HoxA9 and HoxA10 appeared to be regulated at least in part by DNA methylation, as their expression was derepressed by 5 -aza-dC-treatment. Notably, their expression was derepressed not only by Bmil knockout, but also by Dmapl knockdown (Fig. 4B), indicating that Bmil and Dmap1 cooperatively repress these Hox genes. Although HoxD10 was downregulated by both Bmil knockout and Dmapl knockdown (Fig. 4B), this might represent an indirect effect of the Bmil-Dmap1 complex. On the other hand, HoxC13 expression was derepressed only 
by Bmil knockout, and not by 5-aza-dC-treatment or Dmap1 knockdown (Fig. 4B). In this locus, H2A ubiquitination could be most critical in gene silencing as reported (5). Importantly, Dmapl knockdown in MEFs also resulted in the derepressed expression of Bmil targets we identified in hematopoietic cells (Fig. 4C). In contrast to Dmapl knockdown, however, Bmil knockout did not affect the expression of Rnf103 and ClqTr6 in MEFs. The different cell type-specificities of Bmil and Dmap1 in terms of gene silencing could be ascribed to a major role of Rnf110/Me118, another mammalian homologue of Drosophila Psc, in this process in MEFs or are indicative of more general contribution of Dmap1 to gene silencing. 


\section{Discussion}

Bmil has been characterized as an essential molecule in the maintenance of cellular memory. It contributes to the stable gene silencing at least in part by cooperating with Ring1A and Ring1B in H2A-K119 ubiquitination (5). In the present study, we identified a novel Bmil complex operating in gene silencing. Bmil recruits Dmap1 and Dnmt1 to its target loci and forms a ternary complex within the context of the PRC1. As demonstrated at the Bmil target loci we identified in this study, the loss of Bmil-Dmap1 complex was tightly associated with gene reactivation in $\mathrm{Bmil}^{-/}$cells and Dmapl knockdown cells, indicating that Bmi1 cooperates with Dmap1 in the maintenance of gene silencing.

The function of Dmap1 has not yet been fully clarified. It reportedly interacts and colocalizes to DNA replication foci with Dnmt1 throughout the S phase, and also interacts with the transcriptional co-repressor TSG101 (12). The Dmap1-Dnmt1 complex interacts with the p33ING1-Sin3-HDAC complex physically through direct interaction between Dmap1 and p33ING1 at pericentric heterochromatin in the late S phase, and both complexes contribute to the maintenance of pericentric heterochromatin structure (15). In this study, we unveiled a novel aspect of Dmap1 function in gene silencing in collaboration with Bmi1. It has recently been shown that the localization of Bmil to PcG bodies is disrupted when Dnmt1 is depleted by RNAi (16). This raises the possibility that at certain gene loci, Bmi1 and Dmap1-Dnmt1 mutually reinforce each other's recruitment to specific promoters and cooperate to repress transcription. On the other hand, Dmap1 has also been characterized as one of the component molecules of the NuA4b HAT multisubunit complex (17). The functional impact of Dmap1 in this HAT complex remains to be determined.

At the Bmil target loci we analyzed, the knockout of Bmil resulted in a substantial decrease in $\mathrm{H} 2 \mathrm{~A}$ ubiquitination and in a considerable increase in acetylation of H3K9 (Fig. 
3A). The former finding is consistent with an important role for Bmil in $\mathrm{H} 2 \mathrm{~A}$ ubiquitination (5). Regarding the latter finding, it is possible that Bmil affects HATcontaining complex. Or, given that Dnmt1 directly binds HDACs, and that Dnmt1 is tightly associated with histone deacetylase activity in vivo $(12,18)$, the Bmi1-Dmap1Dnmt1 complex might also be connected with histone deacetylation activity.

Long-term gene silencing involves maintenance of proper $\mathrm{CpG}$ methylation patterns and tethering repressive regulators to methylated $\mathrm{CpG}$ dinucleotides, including methyl-CpG binding proteins (MBPs), which contribute to establish and maintain transcriptional repression. It should be noted that most of the Bmil targets we analyzed in this study are DNA methylation-sensitive. In addition, we observed alterd $\mathrm{CpG}$ methylation patterns in $\mathrm{Bmil}^{-/}$cells, which could lead to disruption in gene silencing. Altered DNA methylation patterns in $\mathrm{Bmil}^{-/-}$cells included both local hypomethylation and hypermethylation. The similar dysregulation of the $\mathrm{CpG}$ methylation patterns has been observed at the imprinting gene loci on distal chromosome 7 in embryo deficient for Eed (19), a PRC2 component that makes direct contact with Dnmt1, Dnmt3a and Dnmt3b, like Ezh2 (13). Given that Bmi1 forms a complex with Dnmt1 through Dmap1, it is possible that Bmi1 as well as Eed/Ezh2 controls DNA methyltransferases to precisely propagate DNA methylation patterns of their target loci. Detailed analyses are required to address whether Bmil is really involved in the control of local DNA methylation.

PcG gene silencing is a diversified process that involves both DNA methylation and various chromatin modifications. The way to establish gene silencing should be unique to each gene loci. Our findings provide the Bmi1-Dmap1-Dnmt1 complex as one of the core complexes that govern this process and might be relevant to various gene-silencing processes, such as imprinting, X-chromosome inactivation and heterochromatinization. The biological significance of this complex should be tested. 


\section{Acknowledgements}

We thank Dr. M. van Lohuizen for providing the $B m i 1^{-/-}$mice, Dr. T. Ushijima for the MSRDA protocol, Dr. H. Miyoshi for CS-H1-shRNA-EF-1 $\alpha$-EGFP, Dr. M. Yamashita for his valuable discussion, Dr. H. Hashimoto for kind instruction and H. Tsukui and A. Takahashi for their excellent technical assistance. This work was supported in part by grants from the Ministry of Education, Culture, Sport, Science and Technology, Japan, Core Research for Evolutional Science and Technology (CREST) of Japan Science and Technology Corporation (JST), the Takeda Science Foundation and the Uehara Memorial Foundation (to A.I.). The authors have no conflicting financial interests. 


\section{References}

1. A.H. Lund, M. van Lohuizen, M. Polycomb complexes and silencing mechanisms, Curr. Opin. Cell Biol. 16 (2004) 239-246.

2. A. Kuzmichev, T. Jenuwein, P. Tempst, D. Reinberg D, Different Ezh2-containing complexes target methylation of histone $\mathrm{H} 1$ or nucleosomal histone H3, Mol. Cell 14 (2004) 183-193.

3. A. Kuzmichev, R. Margueron, A. Vaquero, T.S. Preissner, M. Scher, A. Kirmizis, X. Ouyang, N. Brockdorff, C. Abate-Shen, P. Farnham, D. Reinberg, Composition and histone substrates of polycomb repressive group complexes change during cellular differentiation, Proc. Natl. Acad. Sci. U.S.A. 102 (2005) 1859-1864.

4. H. Wang, L. Wang, H. Erdjument-Bromage, M. Vidal, P. Tempst, R.S. Jones, Y. Zhang, Role of histone H2A ubiquitination in Polycomb silencing, Nature 431 (2004) 873878.

5. R. Cao, Y.I. Tsukada Y. Zhang, Role of Bmi-1 and Ring1A in H2A ubiquitylation and Hox gene silencing, Mol. Cell 20 (2004) 845-854.

6. M.E. Valk-Lingbeek, S.W.M. Bruggeman, M. van Lohuizen, Stem cells and cancer: the polycomb connection, Cell 118 (2004) 409-418.

7. A. Iwama, H. Oguro, M. Negishi, Y. Kato, H. Nakauchi, Epigenetic regulation of hematopoietic stem cell self-renewal by polycomb group genes, Int. J. Hematol. 81 (2005) 294-300.

8. A. Iwama, H. Oguro, M. Negishi, Y. Kato, Y. Morita, H. Tsukui, H. Ema, T. Kamijo, Y. Katoh-Fukui, H. Koseki, M. van Lohuizen, H. Nakauchi, Enhanced self-renewal of hematopoietic stem cells mediated by the polycomb gene product, Bmi-1. Immunity $21(2004) 843-851$. 
9. H. Oguro, A. Iwama, Y. Morita, T. Kamijo, M. van Lohuizen, H. Nakauchi, Differential impact of Ink4a and Arf on hematopoietic stem cells and their bone marrow microenvironment in Bmil-deficient mice, J. Exp. Med. 203 (2006) 22472253.

10. M. Masuhara, K. Nagao, M. Nishikawa, M. Sasaki, A. Yoshimura, M. Osawa, Molecular cloning of murine STAP-1, the stem-cell-specific adaptor protein containing PH and SH2 domains. Biochem. Biophys. Res. Commun. 268 (2000) 697703.

11. T. Ushijima, K. Morimura, Y. Hosoya, H. Okonogi, M. Tatematsu, T. Sugimura, M. Nagao, Establishment of methylation-sensitive-representational difference analysis and isolation of hypo- and hypermethylated genomic fragments in mouse liver tumors, Proc. Natl. Acad. Sci. U.S.A. 94 (1997) 2284-2289.

12. M.R. Rountree, K.E. Bachman, S.B. Baylin, DNMT1 binds HDAC2 and a new corepressor, DMAP1, to form a complex at replication foci, Nat. Genet. 25 (2000) 269277

13. E. Vire, C. Brenner, R. Deplus, L. Blanchon, M. Fraga, C. Didelot, L. Morey, A. Van Eynde, D. Bernard, J.M. Vanderwinden, M. Bollen, M. Esteller, L. Di Croce, Y. de Launoit, F. Fuks, The polycomb group protein EZH2 directly controls DNA methylation, Nature 439 (2006) 871-874.

14. N.M. van der Lugt, M. Alkema, A. Berns, J. Deschamps, The Polycomb-group homolog Bmi-1 is a regulator of murine Hox gene expression, Mech. Dev. 58 (1996) 153-164.

15. H. Xin, H. -G. Yoon, P.B. Singh, J. Wong, J. Qin, Components of a pathway maintaining histone modification and heterochromatin protein 1 binding at the pericentric heterochromatin in mammalian cells, J. Biol. Chem. 279 (2004) 9539- 
9546.

16. I. Hernandez-Munoz, P. Taghavi, C. Kuijl, J. Neefjes, M. van Lohuizen, Association of BMI1 with polycomb bodies is dynamic and requires $\mathrm{PRC} 2 / \mathrm{EZH} 2$ and the maintenance DNA methyltransferase DNMT1, Mol. Cell. Biol. 25 (2005) 1104711058.

17. Y. Doyon, W. Selleck, W.S. Lane, S. Tan, J. Cote, Structural and functional conservation of the NuA4 histone acetyltransferase complex from yeast to humans, Mol. Cell. Biol. 24 (2004) 1884-1896.

18. F. Fuks, W.A. Burgers, A. Brehm, L. Hughes-Davies, T. Kouzarides, T, DNA methyltransferase Dnmt1 associates with histone deacetylase activity, Nat. Genet. 24 (2000) 88-91.

19. J. Mager, N.D. Montgomery, F.P. de Villena, T. Magnuson, Genome imprinting regulated by the mouse Polycomb group protein Eed, Nat. Genet. 33 (2003) 502-507. 


\section{Figure legends}

\section{Figure 1. Bmi1 forms a ternary complex with Dmap1 and Dnmt1.}

(A) Mapping of the interaction domains between Bmi1 and Dmap1. Interaction of Bmi1 and Dmap1 was evaluated by the growth on selection plates lacking histidine (HIS-) and by $\beta$-galactosidase activity in a yeast two-hybrid system. Rnf, ring finger domain; HT, helixturn-helix-turn-helix-turn domain; P/S, Proline/Serine-rich domain; SANT, SANT domain. The region of Dmap1 (amino acids 212-422) that interacts with Dnmt1 is indicated by an asterisk. (B) The whole-cell extracts from COS-7 cells transfected with Flag-Bmi1, HADmap1 and Myc-Dnmt1 were immunoprecipitated by anti-Flag antibody (left panel) or anti-HA antibody (right panel). Western blot analysis was performed using the indicated antibodies. (C) In vitro translated HA-Dmap1 or Myc-Dnmt1 was incubated with GST or GST-Bmi1 fusion proteins. (D) Endogenous Dmap1 and Dnmt1 were coimmunoprecipitated with Bmil. The whole-cell extracts from Bmil-transfected NIH3T3 cells were immunoprecipitated with either control IgG or anti-Bmil antibody. Western blot analysis was performed using the indicated antibodies. (E) Co-fractionation of Bmi1, Dmap1 and Dnmt1 on a glycerol gradient. Whole cell lysate of NIH3T3 cells was separated on a $10-50 \%$ glycerol gradient. Fractions were probed with antibodies against Bmi1, Dmap1, Dnmt1 and Ring1b. Fraction number is indicated on the top of the panel. 'In' represents input. An asterisk indicates non-specific band. Thyroglobulin (660kDa) and aldorase $(160 \mathrm{kDa})$ were used as protein markers and were fractionated on a separate gradient that was run in parallel, and fractions containing these proteins are indicated on the top of the panel.

\section{Figure 2. Identification of Bmi1 target loci.}


(A) RT-PCR demonstrated the de-repressed expression of genes related to MS-RDA loci in $\mathrm{Bmil}^{-/-}$lineage-marker immature fetal liver hematopoietic cells (E14.5). Gapdh, glyceraldehyde-3-phosphate dehydrogenase. (B) Reactivation of Bmil target genes by DNA demethylation. MEL cells were treated with 5-aza-dC for 96 hours. RT-PCR analysis was performed on MS-RDA-related genes in 'A'. Hprt, hypoxanthine-Guanine phosphoribosyl transferase. (C) ChIP assays of the Bmil target loci show the association of Bmi1, Dmap1 and Dnmt1 at the ClqTr6 gene locus and the Rnf103 locus, and (D) the Bmi1-Dmap1-Dnmt1 ternary complex formation within the context of the PRC1 at the WDr77 locus in Bmil-overexpressing MEL cells. The top panels are the schematic representations of each gene locus. Asterisks indicate the genomic fragments identified by MS-RDA screening. The genomic regions analyzed cover about $200 \mathrm{bp}$ and are indicated in numbers.

\section{Figure 3. Bmi1 recruits Dmap1 and Dnmt1 to its target loci.}

ChIP assays of the Bmil target loci in $\mathrm{Bmil}^{-/-}$cells demonstrate a significant reduction in Dmap1 and Dnmt1 binding concomitant with the decreased H2A ubiquitination and increased $\mathrm{H} 3 \mathrm{~K} 9$ acetylation. The promoter regions of the indicated gene loci and a distal region of the $W D r 77$ gene locus were analyzed by ChIP using wild-type and $B m i 1^{-/-}$fetal liver cells (E14.5). The promoter regions detected encompass WDr77 regions 4 and 5, ClqTr6 region 3 and Rnf103 regions 3 and 4 depicted in Fig. 4, and the distal region was designed at $4 \mathrm{~kb}$ upstream of the $W D r 77$ ATG.

\section{Figure 4 Hox loci are derepressed in $\mathrm{Bmil}^{-/-}$MEFs and Dmap1 knockdown MEFs.}

(A) Knockdown of the endogenous Dmapl in infected MEFs was confirmed by western blotting. Tubulin served as a loading control. The antibodies used are indicated on the 
Negishi M, et al. 20

left. (B, C) RT-PCR analysis of the Hox genes $(\mathbf{B})$ and the Bmil target genes $(\mathbf{C})$ in $\mathrm{Bmil}^{-}$ /- MEFs, MEFs treated with 5-aza-dC for 96 hours, Dmapl knockdown MEFs and control MEFs infected with shRNA virus against the Luciferase gene. 
A

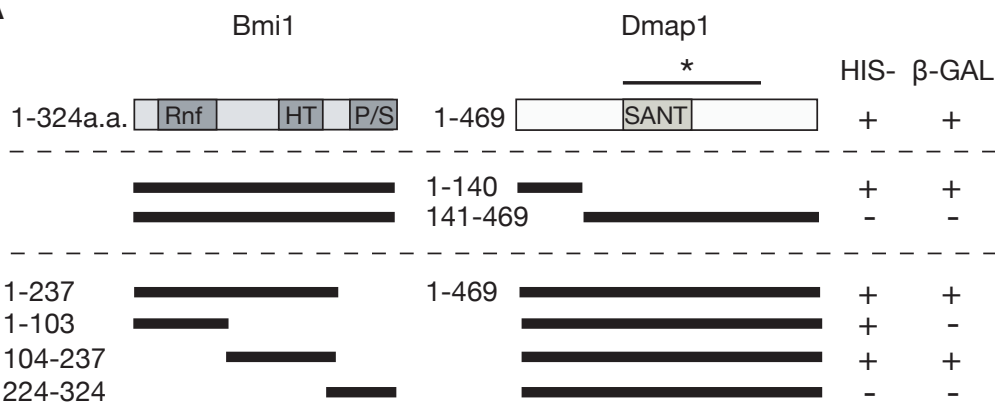

B
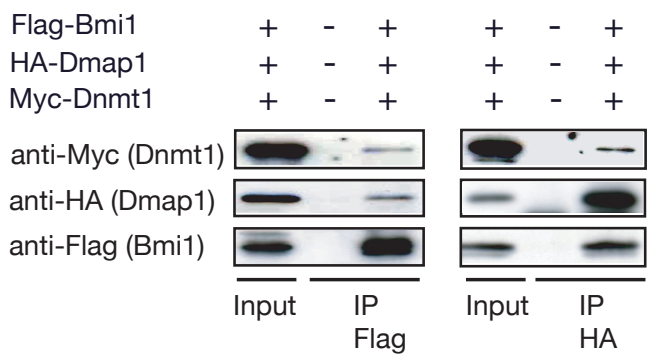

C

D

Input IP IP
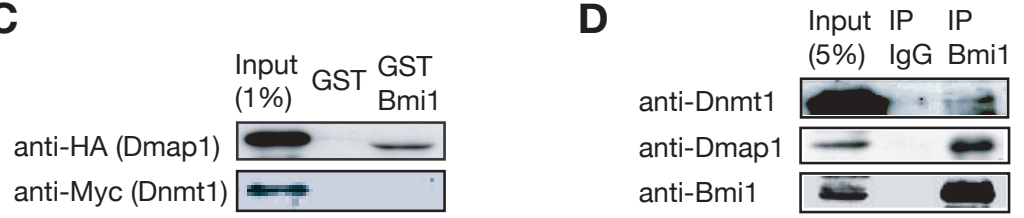

$\mathbf{E}$

$160 \mathrm{kDa}$

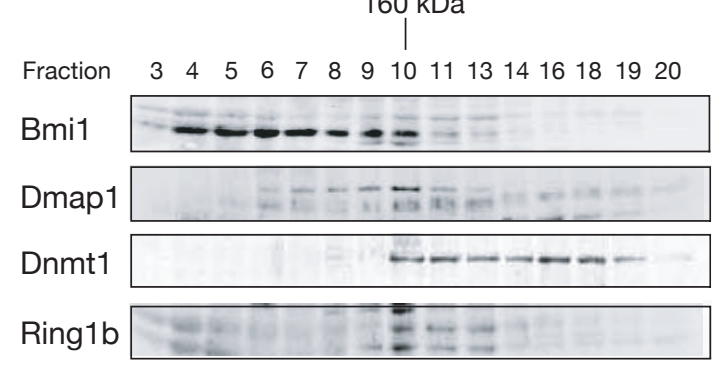

$660 \mathrm{kDa}$

1

$\begin{array}{lllllllllllllll}21 & 22 & 23 & 24 & 26 & 27 & 29 & 30 & 31 & 32 & 33 & 34 & 35 & 36 & \text { In }\end{array}$
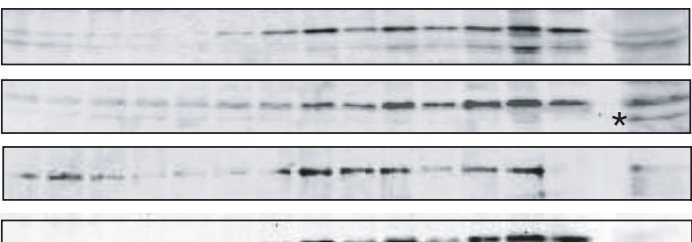
Figure 2 Negishi et al

A

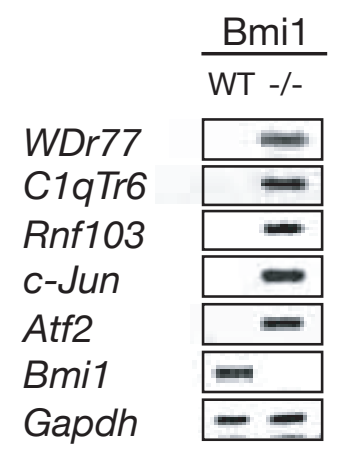

B

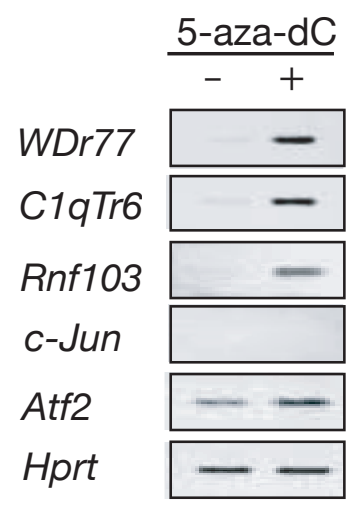

C
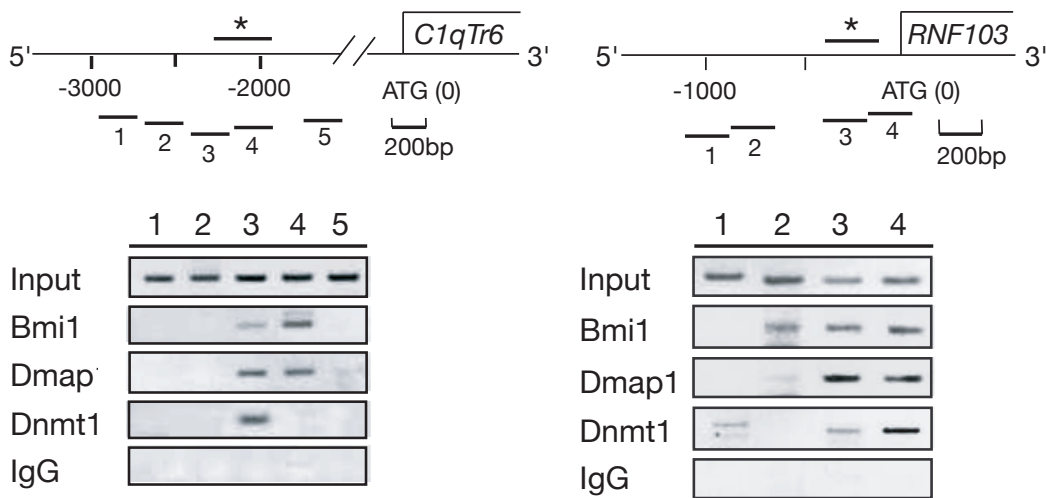

D
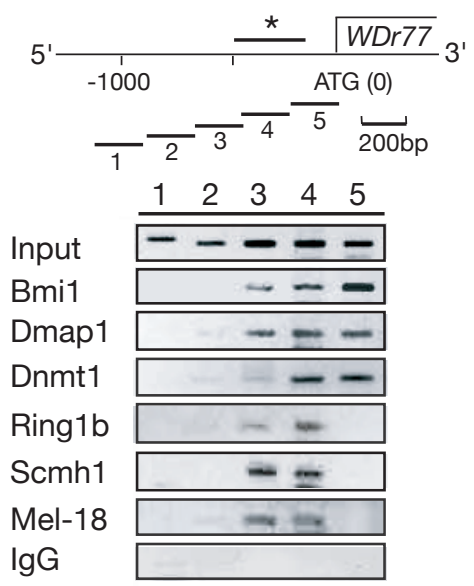
Figure 3 Negishi et al

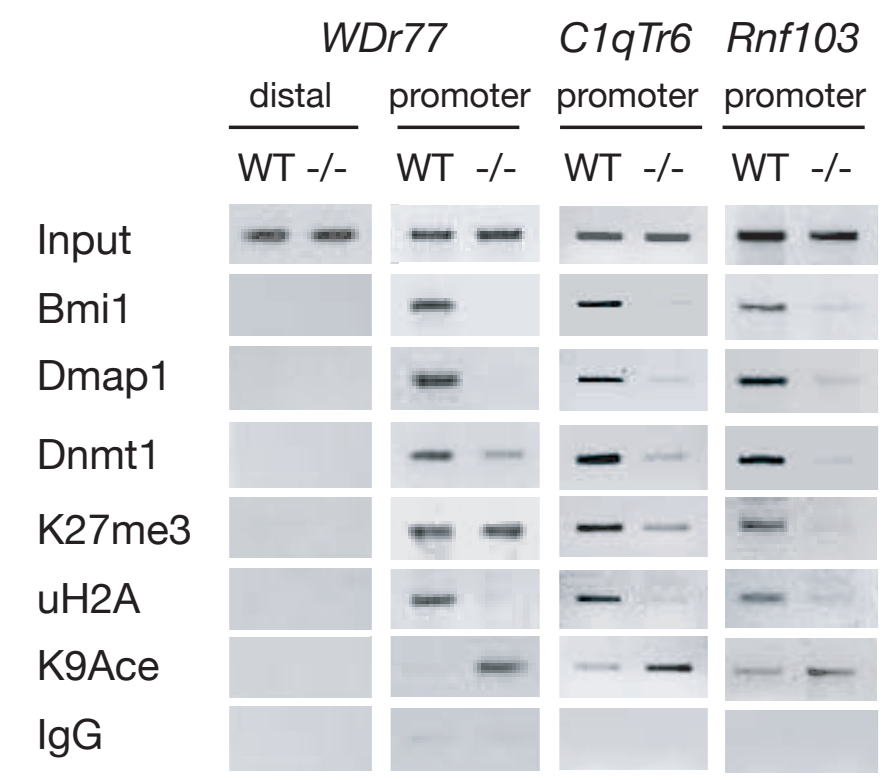


Figure 4 Negishi et al

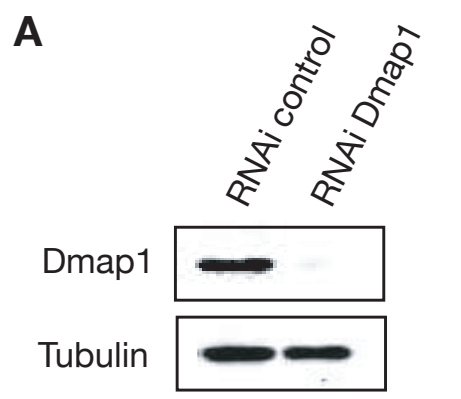

B

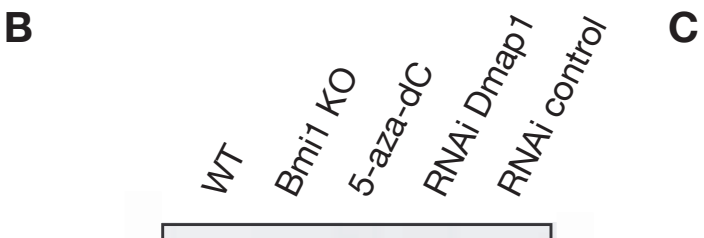

C
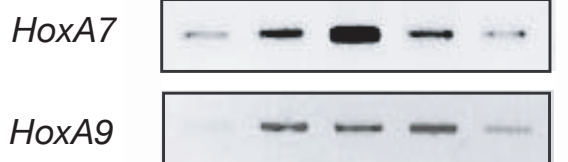

WDr77

HoxA10

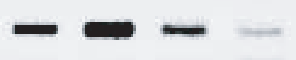

Rnf103

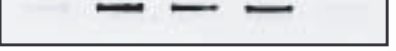

HoxC13

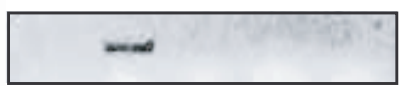

C1qTr6

$\longrightarrow$

HoxD10

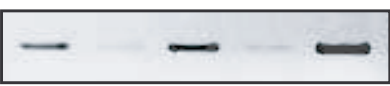

WDr77

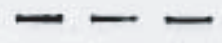

Hprt

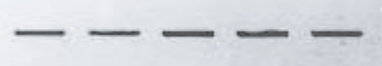

Hprt
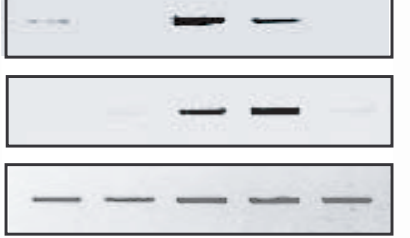